\title{
Desafios e potencialidades envolvidos na prevenção de doenças diarreicas junto à população indígena em Roraima
}

\author{
Challenges and potentialities involved in the prevention of diarrh eal diseases with the \\ indigenous population in Roraima
}
Retos y potencialidades de la prevención de las enfermedades diarreicas con la población indígena de Roraima

Thalyta Ketlen de Melo Oliveira ${ }^{1}$, Tárcia Millene de Almeida Costa Barreto ${ }^{1}$, Maria Bárbara de Magalhães Bethonico', Paulo Sérgio da Silva1, Fabiano Barreto ${ }^{1}$, Ana Paula Barbosa Alves ${ }^{1}$, Kenisson Oliveira Rodrigues². Fabrício Barreto ${ }^{1 *}$.

\section{RESUMO}

Objetivo: Analisar os desafios e as potencialidades envolvidas nas ações de cuidado preventivo para doenças diarreicas realizadas pela equipe de enfermagem junto a população indígena. Métodos: Estudo descritivo com abordagem qualitativa. Participaram da investigação 11 profissionais de enfermagem não indígenas. Os profissionais foram investigados por questionário quanto aos desafios e potencialidades envolvidos nas atuações em áreas indígenas. A análise dos dados foi realizada segundo o conteúdo teorizado por Laurence Bardin. O estudo foi aprovado por Comitê de Ética em Pesquisa. Resultados: Destacam-se as estruturas locais relacionadas com a água para consume e a higiene corporal como os principais desafios enfrentados no combate as diarreias. Do ponto de vista da potencialidade a educação em saúde se mostrou como uma potente estratégia para cuidar da população indígena suscetível ao desenvolvimento de diarreias. Conclusão: Os quadros diarreicos foram associados a precarização sanitária onde destaca-se o sistema de distribuição de água que atende as comunidades indígenas como um desafio para cuidar. Em contrapartida o compromisso da equipe de enfermagem com a educação em saúde foi analisado como uma potencialidade na prevenção e tratamento das doenças diarreicas junto a população indígena.

Palavras-chave: Saúde indígena, Diarreia, Prevenção, Enfermagem.

\section{ABSTRACT}

Objective: To analyze the challenges and potential involved in preventive care actions for diarrheal diseases carried out by the nursing staff with the indigenous population. Methods: Descriptive study with a qualitative approach. Eleven non-indigenous nursing professionals participated in the investigation. The professionals were investigated through a questionnaire regarding the challenges and potential involved in acting in indigenous areas. Data analysis was performed according to the content theorized by Laurence Bardin. The study was approved by the Research Ethics Committee. Results: Local structures related to drinking water and personal hygiene stand out as the main challenges faced in combating diarrhea. From the point of view of potential, health education proved to be a powerful strategy to take care of the indigenous population susceptible to the development of diarrhea. Conclusion: Diarrheal conditions were associated with precarious sanitary conditions where the water distribution system that serves indigenous communities stands out as a challenge to take care of. On the other hand, the commitment of the nursing team to health education was analyzed as a potential in the prevention and treatment of diarrheal diseases among the indigenous pop ulation.

Key words: Indigenous health, Diarrhea, Prevention, Nursing.

1 Universidade Federal de Roraima (UFRR), Boa Vista - RR. *E-mail: fabricio.barreto@ufrr.br

2 Distrito Sanitário Especial Indígena do Leste de Roraima (DSEI/LESTE), Boa Vista - RR.

SUBMETIDO EM: 12/2021 


\section{RESUMEN}

Objetivo: Analizar los desafíos y potencialidades de las acciones de atención preventiva de enfermedades diarreicas que realiza el personal de enfermería con la población indígena. Métodos: Estudio descriptivo con abordaje cualitativo. Participaron de la investigación once profesionales de enfermería no indígenas. Los profesionales fueron investigados a través de un cuestionario sobre los desafíos y potencialidades que implica actuar en áreas indígenas. El análisis de los datos se realizó de acuerdo con el contenido teorizado por Laurence Bardin. El estudio fue aprobado por el Comité de Ética en Investigación. Resultados: Las estructuras locales relacionadas con el agua potable y la higiene personal se destacan como los principales desafíos enfrentados en el combate a la diarrea. Desde el punto de vista de la potencialidad, la educación para la salud resultó ser una poderosa estrategia para atender a la población indíg ena susceptible al desarrollo de diarreas. Conclusión: Las condiciones diarreicas se asociaron con precarias condiciones sanitarias donde el sistema de distribución de agua que atiende a las comunidades indígenas se destaca como un desafío a cuidar. Por otro lado, se analizó el compromiso del equipo de enfermería con la educación en salud como potencial para la prevención y tratamiento de las enfermedades diarreicas en la población indígena.

Palabras clave: Salud indígena, Diarrea, Prevención, Enfermería.

\section{INTRODUÇÃO}

A população indígena sofreu ao longo dos anos interferências na sua dinâmica de vida, sobretudo nos aspectos culturais, ambientais e demográficos. Estas interferências influenciaram significativamente seus determinantes de saúde, pois expuseram este grupo a novos patógenos antes desconhecidos pela comunidade, o que provocou epidemias de doenças e levaram a morte muitos indígenas (MAZZUCCHETTI L, et al., 2014). Nesse contexto, destaca-se a diarreia compreendida como uma doença preocupante nos povos indígenas, pois apesar de ser tratável correspondem a uma das principais doenças infecciosas que os atingem; em muitos casos evoluindo ao óbito, principalmente, de crianças menores de 5 anos (PINTO MV e ZATTI CA, 2018).

Dentre os fatores de riscos atribuídos às principais causas dessa infecção estão a ingestão de água sem tratamento adequado, precariedade de saneamento básico com esgoto a céu aberto e higiene duvidosa, além de consumo de frutas, hortaliças e quaisquer outros alimentos sem saber a procedência do preparo e armazenamento; sendo a população indígena suscetível a estas situações pelas condições de habitação em que faltam recursos de infraestrutura e atendimento à saúde adequados (GENÉSIO KCS, 2017; MINISTÉRIO DA SAÚDE, 2019a).

De maneira peculiar os Distritos Sanitários Especiais Indígenas (DSEls), interligam as ações de saúde indígena aos demais do país, e realizam o atendimento a esta população, por meio da implantação de Polos Base localizados nas aldeias, tornando-o como primeira referência de atendimento, com o objetivo de promover ações protagonizadas por Equipes Multidisciplinares de Saúde Indígena (EMSI) na melhoria das condições de saúde (MINISTÉRIO DA SAÚDE, 2019b).

Por meio da atuação dos DSEls é possível conhecer os problemas de saúde, identificar seus determinantes, planejar, organizar, implementar, monitorar e avaliar as ações a serem feitas na população indígena, além de conhecer as principais comorbidades que os atingem (MINISTÉRIO DA SAÚDE, 2017; SOUSA MCO, et al., 2007).

A equipe de enfermagem precisa estar disposta para atuar na saúde indígena, conseguir identificar situações de risco e atuar preventivamente, em conjunto com os demais profissionais que atuam nas mais variadas ações e programas de saúde, além disso é fundamental a compreensão do processo saúde-doença de forma estendida, incluindo o aspecto étnico-cultural e sua constante busca por atualizações e novos conhecimentos. Mediante o exposto, este estudo teve por objetivo: analisar os desafios e as potencialidades envolvidas nas ações de cuidado preventivo para doenças diarreicas realizadas pela equipe de enfermagem a população indígena. 


\section{MÉTODOS}

Trata-se de uma pesquisa descritiva com abordagem qualitativa desenvolvida com profissionais da enfermagem não indígenas na sede do Distrito Sanitário Especial Indígena Leste de Roraima (DSEI-Leste de Roraima) localizado na cidade de Boa Vista-RR. Participaram do estudo 11 profissionais que atuam diretamente nas comunidades indígenas da área de interesse. Para manter o sigilo da pesquisa, os participantes foram identificados como $P+$ Numeração de 1 a 11 , sendo $P=$ Profissionais.

A pesquisa foi realizada na sede do Distrito Sanitário Especial Indígena Leste de Roraima (DSEI-Leste de Roraima) localizado na cidade de Boa Vista-RR. O DSEI-Leste de Roraima, que abrange os municípios Boa Vista, Alto Alegre, Amajari, Bonfim, Cantá, Normandia, Pacaraima, Uiramutã, São João da Baliza, São Luís e Caroebe. Os povos indígenas localizados ao Leste de Roraima pertencem às etnias Macuxi, Wapichana, Ingarikó, Patamona, Taurepang, Sapará e Wai-Wai, onde existem 323 aldeias. Para fins assistenciais estão divididos em 34 pólos-base e onze regiões geográficas - Serras, Surumu, Baixo Cotingo, Raposa, Amajarí, Taiano, Murupú, Serra da Lua, Ingarikó e Wai-Wai. Estão distribuídos em 32 Terras Indígenas.

A seleção ocorreu por conveniência mediante aceite na participação da pesquisa. Quanto aos critérios de seleção: foram incluídos profissionais de enfermagem não indígenas que atuavam em comunidades indígena do DSEI-Leste de Roraima no período de julho e agosto de 2020. E excluídos os profissionais de enfermagem indígenas, os que não trabalhavam na área e ainda os que se encontravam de férias, tratamento de saúde ou af astamento por quaisquer motivos.

Devido ao período de pandemia e as restrições de isolamento social, a coleta de dados ocorreu por meio de um questionário. Com auxílio da coordenação de enfermagem do DSEI-Leste, foram entregues cópias dos Termos de Consentimento Livres e Esclarecidos (TCLE) e do questionário a serem preenchidos. Após a assinatura do TCLE pelos participantes, em duas vias, onde uma permaneceu com o participante e a outra com o pesquisador, iniciaram as coletas de informações pelos questionários, tendo o contato da pesquisadora para esclarecimentos de qualquer dúvida que houvesse. Conforme os questionários foram sendo preenchidos, a coordenação os guardava em um envelope, que era buscado pela pesquisadora quinzenalmente.

Cabe salientar que no plano ético-investigativo foi encaminhado o termo de anuência para o coordenador distrital do DSEI-Leste de Roraima com o intuito de receber o consentimento para realização da pesquisa. Após o consentimento foi enviado o projeto para avaliação do Comitê de Ética e Pesquisa (CEP), da instituição proponente, que concedeu a autorização da pesquisa, conforme Resolução do Conselho Nacional de Saúde no466/2012, tendo sido aprovado pelo CAEE: 30066620.7.0000.5302, em 16/7/2020. Somente após todas as aprovações foram iniciados os contatos com os participantes do estudo, sendo solicitado a assinatura do Termo de Consentimento Livre e Esclarecido (TCLE), em duas vias, onde uma permaneceu com o participante e a outra com o pesquisador, garantindo a confiabilidade do estudo, a privacidade, sigilo das informações e o anonimato dos integrantes da pesquisa. Destaca-se que ao final do estudo, houve o compromisso de divulgar os resultados de forma acessível ao grupo pesquisado, como forma de contribuição.

Por fim, o presente estudo não trabalhou com quantificação numérica, mas pelo modelo analítico de conteúdo de Bardin L (2016), que prevê a categorização dos achados mediante a repetição do discurso, ou seja, o encontro dos pontos em comum nos registros foi organizado em categorias analíticas, que se refere três diferentes etapas existentes na técnica para organização da análise, sendo: a pré-análise (organização do material); exploração (classificação ou categorização e codificação); por fim, a interpretação dos resultados.

\section{RESULTADOS E DISCUSSÃO}

Esta unidade foi estruturada em três blocos temáticos a partir da coleta de dados, sendo analisados em três eixos temáticos que envolve o conteúdo estudado: 1) caracterização dos participantes do estudo, 2) água e higiene: desafios na prestação de cuidados preventivos de enfermagem nas diarreias junto à população indígena, 3) potencialidades na prevenção das doenças diarreicas com a população indígena. 
No que se refere as temáticas 2 e 3, que estão relacionadas as perguntas abertas, as repostas aos questionários foram em sua maioria muito objetivas, e demasiadamente curtas, e dessa forma a análise ocorreu a partir de palavras que se repetiam nos discursos e as falas mencionadas foram selecionadas a partir do aprofundamento das respostas.

\section{Caracterização dos participantes do estudo}

Participaram do estudo 11 (onze) profissionais de enfermagem, sendo 6 (seis) do sexo feminino (54,5\%) e 5 (cinco) do sexo masculino (45,5\%), com idade entre 33-43 anos, em média 36,6 anos, com média de 8,9 anos de formação e média de 4,7 anos de atuação no DSEI-Leste de Roraima. Dos entrevistados apenas 4 (três) possuem algum tipo de especialização (36,3\%), e destes, somente 1 (um) com informações de especialização em saúde indígena. Sobre o contato com saúde indígena antes de atuar no DSEI-Leste, somente 1 (um) já tinha tido esta experiência (9\%). Em relação a área de atuação, foram identificados 5 (cinco) regiões, em sua maioria da região de São Marcos (36,3\%).

Houve uma prevalência no sexo feminino no estudo e Machado MH (2017) denota a tradição que ainda há na profissão, correspondendo ao estudo do perfil da enfermagem no Brasil que analisou as tendências e perspectivas da enfermagem no contexto socioeconômico e geopolítico. Mas não só o sexo apresenta uma tendência, a idade média dos participantes é de 36,6 anos, semelhante ao o estudo realizado no Amazonas, envolvendo o Distrito Sanitário Especial Indígena Alto Rio Negro, sobre o perfil de enfermeiros (as) que atuam na saúde indígena e não indígena, em que a média de idade era de 35 anos, assim como também o tempo de formação é semelhante, sendo de 7,3 anos, e se diferem quando comparados ao tempo de atuação envolvendo a população indígena, sendo em média 2 anos (ROCHA ESC, et al., 2018).

No que tange à formação Fernandes GCM, et al. (2013) reportam quanto a formação complementar, o fato de menos da metade dos entrevistados possuírem uma especialização na área de atuação, que denota uma tendência diminuído pelos profissionais por qualificação, acarretando uma percepção de cuidado comprometida para a sensibilidade cultural e pela busca por estratégias que lidam com as diferenças relacionadas a assistência.

\section{Água e higiene: desafios na prestação de cuidados preventivos de enfermagem nas diarreias junto a população indígena}

Quando indagados qual seria a principal causa de diarreia no seu local de atuação, fo i possível distinguir duas palavras-chave: água e higiene, e diante da contextualização destas, a água é referida como contaminada, não potável e de difícil acesso, e a higiene é direcionada tanto aos alimentos quanto a corporal como inadequada e precária:

"A principal causa é a falta de higienização das mãos, água contamina e alimentos contaminados, não há local adequado" (P9).

“... falta de higiene e água potável limpa é de difícil acesso" (P3).

“... água, alimentos contaminados por parasitas" (P8).

“... água não tratada, falta de saneamento básico" (P5).

A partir dos registros observa-se que a potabilidade da água é um dos principais desafios, sendo muito questionada quanto a sua qualidade. Nesse sentido, no estudo desenvolvido por Pena JL e Heller L (2008), no período de 2000 a 2003, sobre saúde e saneamento na população Xakriabá, foi evidenciado que a rede de abastecimento de água é insuficiente para atender em condições adequadas, visto que no estudo, apenas 22,2\% dos domicílios apresentaram ausência de coliformes totais e Escherichia coli., destacando que 83,3\% mostraram que a água era imprópria para o consumo humano. Os resultados ainda mostram a relação entre as variáveis incidência de diarreia versus porcentagem de população infantil abastecida com rede geral e chaf ariz, no período de 2001 a 2002, que correspondem aos achados científicos sobre o ef eito do saneamento básico sobre os indicadores de saúde.

Além disso, Coimbra JR CEA (2014) corrobora em seus estudos, no período de 2008 a 2009, onde visitou 113 aldeias e constatou que na região Norte há uma distinção das demais regiões quanto a predominância 
na origem da água utilizada para beber, vindo direta de rios, igarapés, lagos ou açudes sem nenhum tratamento. Silva RP e Dourado DG (2019) recordam que a ausência de tratamento da água e saneamento básico está diretamente relacionada com doenças infecciosas e parasitárias, e afirmam que estas permanecem como importante causa de morbimortalidade dos povos indígenas no Brasil, destacando a importância de educação em saúde.

Não só a infraestrutura, mas a implementação de hábitos de higiene é um obstáculo, como mencionado nas narrativas supracitadas, e segundo a percepção dos participantes, a adesão aos cuidados tanto de higiene como a adesão ao tratamento e também a implementação de hábito preventivos estão relacionados a cultura indígena, como relata a seguir:

“...cuidado com a água” (P1).

“Cultura” (P2).

"Fazer com o que eles tomem a medicação (antiparasitários), se nega a tomar ou não toma” (P3).

"A população seguir as orientações de cuidado com a água e alimentos" (P4).

"Cultura e a falta do seguimento das orientações que passamos sobre higiene e lavagem das mãos" (P7).

"Conscientizar pacientes e familiares da importância de cozinhar bem os alimentos, ferver a água ou usar cloro, onde o mesmo nem sempre fazem isso” (P8).

“... são da aceitação dos pacientes quanto as orientações e alguns lugares que falta estrutura de saneamento básico como água encanada e fossa” (P9).

"Falta de água potável e da adesão as orientações de higienização dos alimentos" (P10).

Fernandes MNF e Simpson CA (2016), salientam que a educação em saúde realizada pela equipe de enfermagem é essencial aos povos indígenas, demonstrando que a sensibilidade a cultura é primordial para uma ef etiva assistência, visto que a prestação de cuidado em algumas situações torna-se uma espécie de negociação entre a medicina ocidental e a medicina tradicional. Em sintonia, Andrade GASCR e Terra MF (2018), revelam que dentre as maiores dificuldades referidas pelos profissionais de saúde está a falta de preparo com relação à cultura e costumes próprios da população indígena. E que a dificuldade na comunicação prejudica a implementação dos programas de saúde que subsidiam a assistência preventiva voltada aos indígenas.

Para que se estabeleça um método terapêutico é essencial que os profissionais busquem entender o que está relacionado a doença e a cura aos povos nativos da região em qual está inserido, uma vez que o processo saúde-doença é estreitamente relacionado ao delineamento cultural de cada indivíduo (SILVA NC, ET AL., 2003).

A causa, os sintomas, o prognóstico, a cura e a prevenção estão associadas a um complexo ritual de crenças e práticas de saúde, que dão sentido aos acontecimentos envolvendo os indivíduos e seus familiares e servem para a tomada de decisões terapêuticas a serem seguidas. Esse reconhecimento é essencial para que ações de cuidado estabelecido nos serviços de saúde seja pautado pelo respeito às diferentes formas de expressão e de comportamento diante dos agravos e assim agregar com a medicina tradicional, e melhorar adesão aos cuidados estabelecidos conforme a assistência biomédica (COIMBRA JR CEA, 2014).

\section{Educação em saúde: potencialidades na prevenção das doenças diarreicas em populações indígenas}

No que diz respeito as potencialidades envolvidas no cuidado preventivo para diarreias com a população indígena, Martins JCL (2017), destaca a notoriedade importância de promover a prevenção a partir das oportunidades de educação em saúde. Em quaisquer circunstâncias, seja antes, durante ou depois dos episódios diarreicos persistentes, visando a prevenção desta doença, como também de outras que se 
manifestam como consequência dela, ou tem como fatores de risco a higienização de alimentos e corporal precárias, e o consumo de água inadequado. Neste contexto destacam:

"Orientar quanto ao uso de água limpa, lavagens das mãos e higienização dos alimentos durante palestras e consultas de enfermagem" (P1).

"Orientações em palestras sobre lavagem dos alimentos, uso de água potável, lavagem das mãos (como lavar)" (P3).

"Educação em saúde voltada ao cuidado com a lavagem dos alimentos, ferver a água de consumo, lavas as mãos" (P4).

"Seguir as orientações do protocolo AIDIPI: palestras, orientações sobre alimentação, aleitamento materno exclusivo e higiene” (P8).

“...palestras e orientações de higienização das mãos, higienização e cuidados no preparo dos alimentos, água potável fervida ou filtrada, aleitamento materno exclusivo até os 6 meses, evitar excesso de açucares e gorduras” (P9).

A prática de educação em saúde demanda uma necessidade de capacitação profissional e sua ef etividade está associada com a interação do profissional com o paciente, e forma que essa interação é estabelecida a partir das características pessoais de cada indivíduo. O estudo de Viana JA, et al. (2020), denotam a educação em saúde como uma prática transformadora para melhoria da qualidade da assistência à saúde em aldeias indígenas, e ainda reconhece como potencialidade da enfermagem o comprometimento dos profissionais, com as atividades de saúde voltadas aos cumprimentos dos princípios do Sistema Único de Saúde (SUS).

A proximidade das equipes de enfermagem com a população indígena favorece a vigilância direta na realização do cuidado, assim como o diagnóstico das deficiências na execução das práticas de cuidado ensinadas, possibilitando adequação na forma de repassar as orientações (FERNANDES GCM, et al., 2013). Neste contexto, Silva NC, et al. (2003), lembram que a educação em saúde é essencial, assim como a realização de orientação e demonstrações de higiene corporal, mutirões de limpeza ambiental em conjunto com os indígenas e o desenvolvimento de um trabalho educativo contínuo das atividades com o agente indígena de saúde.

Ao descreverem as ações orientadoras de cuidado atribuídas a prevenção da diarreia, destacou-se a palavra soro, atribuído ao de tratamento e a prevenção de agravamento a partir da hidratação dos indivíduos, além das ações de educação em saúde como as orientações, e pouco mencionado o uso de medicamentos:

"Reidratação oral (soro de hidratação oral), orientação para ingestão de legumes, água, chá, remédios da medicina tradicional, tratamento medicamentoso, se necessário, dependendo do quadro de desidratação" (P9)

“...soro oral, e dependendo do estado de evolução, medicamentos” (P1).

“...soro e orientação para ingesta hídrica” (P4).

"Soro de hidratação, se der febre antibiótico + verminose" (P5).

"AIDIPI: soro oral e ingestão de líquidos para prevenir desidratação grave" (P8).

"Hidratação com soro, água potável, e insistência na educação em saúde durante a consulta de enfermagem" (P10).

"Hidratação oral e medicação para cortar a diarreia" (P11).

Entre as complicações das doenças diarreicas destaca-se a desidratação, levando a situações graves e com isso o estado de hidratação deve ser uma das primícias a serem tomadas na abordagem do quadro diarreico o quanto antes (GENÉSIO KCS, 2017). Outros estudos, como de Brandt KG, et al. (2015) apontam que a hidratação venosa vem sendo utilizada desde o princípio do último século, mas o grande aliado, o soro de reidratação oral, foi utilizado pela primeira vez na década de setenta, como a principal ferramenta no combate aos efeitos de uma diarreia infecciosa grave, mostrando-se extremamente eficaz na redução da 
morbimortalidade por esses agravos e tornando-se incontentável os seus benefícios. O próprio Ministério da Saúde adota a estratégia de Atenção Integrada às Doenças Prevalentes na Infância de crianças de dois meses a cinco anos de idade (Aidpi Criança), como estratégia à promoção, à prevenção e ao tratamento dos problemas infantis mais frequentes, dentre eles a diarreia (MINISTÉRIO DA SAÚDE, 2017).

\section{CONCLUSÃO}

Com a certeza do inacabado foi possível traçar análises que tocam os fatores envolvidos na execução de ações preventivas realizadas pela equipe de enfermagem às doenças diarreicas junto a população indígena. Sim, se faz cada vez mais necessário olhar para os quadros diarreicos associados a precarização da assistência sanitária e qualidade da água consumida pelos indígenas, elementos desafiadores na prestação do cuidado. Certamente, a carência de um sistema de distribuição de água de qualidade, que atenda todas as comunidades comprometeu os hábitos de higienização e favoreceu a incidência de casos de doenças diarreicas por veiculação hídrica. Do ponto de vista das potencialidades a educação em saúde desenvolvida pela equipe de enfermagem mostrou-se como um potente elemento de reconhecimento de necessidades e intervenções em saúde junto à população indígena em risco para o desenvolvimento de diarreias e suas complicações.

\section{REFERÊNCIAS}

1. ANDRADE GASCR, TERRA MF. Assistência de enfermagem à população indígena: um estudo bibliográfico. Rev. Arq. Med., 2018;63 (2) 100-104.

2. BARDIN L. Análise de Conteúdo. 1a ed. São Paulo: Edições 70, 2016;141 p.

3. MINISTÉRIO DA SAÚDE. Dados de doenças diarreicas agudas. Brasília: Ministério da Saúde, 2019a. Disponível em: https://www.gov.br/saude/pt-br/assuntos/boletins-epidemiologicos/por-assunto. Acessado em: 20 de novembro 2019.

4. MINISTÉRIO DA SAÚDE. Guia de Vigilância em Saúde: volume único. 2019b. Disponível em: https://www.vs.saude.ms.gov.br/wp-content/uploads/2021/03/guia_vigilancia_saude_3ed.pdf. Acessado em: 19 de janeiro de 2020.

5. MINISTÉRIO DA SAÚDE. Sistema de Informação da Atenção à Saúde Indígena (SIASI). 2017. Disponível em: http://www.saude.gov.br/saude-indigena/gestao/siasi. Acessado em:18 de novembro de 2019.

6. BRANDT KG, et al. Diarreia aguda:manejo baseado em evidências. J. de Pediatria, 2015; 91 (6): 36-43.

7. COIMBRA JR CEA. Saúde e povos indígenas no Brasil: reflexões a partir do I Inquérito Nacional de Saúde e Nutrição Indígena. Rev. Cad. de Saúde Pública, 2014;30(4): 855-859.

8. FERNANDES GCM, et al. Percepcõos das equipes de saúde da família sobre o cuidado profissional às crianças indígenas. Rev. C. Cuidado e Saúde, 2013;12 (1): e13654.

9. FERNANDES MNF, SIMPSON CA. Saúde indígena: experiência de enfermagem com a etnia Munduruku. Biblioteca Lascasas, 2016; 12 (2): 01-09.

10. GENÉSIO KCS. Fatores de risco nas habitações e a ocorrência de diarreias em crianças assistidas pela Estratégia Saúde Família de llha de Guaratiba, Rio de Janeiro (RJ). Dissertação (Mestrado em Saúde Pública) - Programa de pós-graduação em Saúde Pública. Escola Nacional de Saúde Pública Sérgio Arouca. Fundação Oswaldo Cruz, Rio de Janeiro, 2017;98 p.

11. MACHADO MH. Perfil da enfermagem no Brasil: relatório final:Brasil./ coordenado por Maria Helena Machado. Rio de Janeiro: NERHUS - DAPS - ENSP/Fiocruz, 2017;748 p.

12. MARTINS JCL. O trabalho do enfermeiro na saúde indígena: desenvolvendo competências para atuação no contexto intercultural. Dissertação (Mestrado em Ciências) - Programa de Pós-Graduação em Saúde Pública, Universidade Federal de São Paulo, São Paulo, 2017;174 p.

13. MAZZUCCHETTI L, et al. Incidência de síndrome metabólica e doenças associadas na população indígena Khisêdjê do Xingu, Brasil Central, no período de 1999-2000 a 2010-2011. Cad. de Saúde Pública, 2014;30 (1): 01-11.

14. PENA JL, HELLER L. Saneamento e saúde indígena:uma avaliação na população xakriabá, Minas Gerais. Rev. de Engenharia Sanitária Ambiental, 2008; 13 (01): 63-72.

15. PINTO MV, ZATTI CA. Adoecimento Indígena: Aspectos referentes à morbimortalidade indígena. Rev. Brazilian J. of Surgery and Clinical Research, 2018;24 (02): 106-111.

16. ROCHA ESC, et al. Perfil de enfermeiros (as) que atuam na saúde indígena e não indígena. Rev. Ciências Cuidado e Saúde, 2018: 17 (4): e45195.

17. SILVA RP, DOURADO DG. Saneamento e saúde em terras indígenas. Rev. Tellus, 2019, 19 (40): 103-122

18. SILVA NC, et al. Enfermagem em saúde indígena: aplicando as diretrizes curriculares. Rev. Brasileira de Enfermagem, 2003;56(4):388-391.

19. SOUSA MCO, et al. Sistema de Informação da Atenção à Saúde Indígena (SIASI): criação, estrutura e funcionamento. Rev. Cad. de Saúde Pública, 2007; 23 (4) 853-861.

20. VIANA JA, et al. A atuação do enfermeiro na saúde indígena: uma análise integrativa da literatura. Brazilian J. of health Review, 2020;3 (2): 2113-2127. 